\section{Original Article}

Check for updates

\section{OPEN ACCESS}

Received: Jan 30, 2017

Revised: Mar 25, 2017

Accepted: Apr 5, 2017

Correspondence to

Sang-Yoon Park

Gynecologic Cancer Branch and Center

for Uterine Cancer, Research Institute and

Hospital, National Cancer Center, 323 Ilsan-ro,

Ilsandong-gu, Goyang 10408, Korea.

E-mail: parksang@ncc.re.kr

Copyright (c) 2017. Asian Society of

Gynecologic Oncology, Korean Society of

Gynecologic Oncology

This is an Open Access article distributed

under the terms of the Creative Commons

Attribution Non-Commercial License (https://

creativecommons.org/licenses/by-nc/4.0/)

which permits unrestricted non-commercial

use, distribution, and reproduction in any

medium, provided the original work is properly

cited.

ORCID

Myong Cheol Lim

https://orcid.org/0000-0001-8964-7158

Yong Jung Song

https://orcid.org/0000-0002-6103-2466

Sokbom Kang

https://orcid.org/0000-0002-8428-9555

Sang-Yoon Park

https://orcid.org/0000-0001-8325-6009

Conflict of Interest

No potential conflict of interest relevant to this article was reported.

\section{Survival outcomes after extensive} cytoreductive surgery and selective neoadjuvant chemotherapy according to institutional criteria in bulky stage IIIC and IV epithelial ovarian cancer

\author{
Myong Cheol Lim, ${ }^{1,4,5,6}$ Heong Jong Yoo, ${ }^{4,7}$ Yong Jung Song, ${ }^{4,8}$ Sang-Soo Seo, ${ }^{4}$ \\ Sokbom Kang, ${ }^{2,4,6}$ Sun Ho Kim, ${ }^{4}$ Chong Woo Yoo, ${ }^{4}$ Sang-Yoon Park ${ }^{3,4}$
}

'Cancer Healthcare Research Branch, Research Institute and Hospital, National Cancer Center, Goyang, Korea. ${ }^{2}$ Precision Medicine Branch, Research Institute and Hospital, National Cancer Center, Goyang, Korea. ${ }^{3}$ Common Cancer Branch, Research Institute and Hospital, National Cancer Center, Goyang, Korea. ${ }^{4}$ Center for Uterine Cancer, Research Institute and Hospital, National Cancer Center, Goyang, Korea. ${ }^{5}$ Center for Clinical Trials, Research Institute and Hospital, National Cancer Center, Goyang, Korea. ${ }^{6}$ Department of Cancer Control and Population Health, Graduate School of Cancer Science and Policy, National Cancer Center, Goyang, Korea.

${ }^{7}$ Department of Obstetrics and Gynecology, Chungnam National University Hospital, Chungnam, Korea. ${ }^{8}$ Department of Obstetrics and Gynecology, Pusan National University Yangsan Hospital, Yangsan, Korea.

\section{ABSTRACT}

Objective: To investigate the survival outcomes in patients with bulky stage IIIC and IV ovarian cancer, treated by primary debulking surgery (PDS) and selective use of neoadjuvant chemotherapy (NAC) according to institutional criteria.

Methods: Medical records for advanced ovarian cancer patients who were treated at National Cancer Center (NCC) between December 2000 and March 2009 were retrospectively reviewed in the comprehensive cancer center. Bulky stage IIIC and IV ovarian cancer cases were included. Current NCC indication for NAC is determined based on patients' performance status and/or computerized tomography (CT) findings indicating difficult cytoreduction. After NAC, all traces of regressed metastatic ovarian cancer, potentially including chemotherapy-resistant cancer cells, were surgically removed.

Results: Of the 279 patients with bulky stage IIIC and IV, 143 (51\%) underwent PDS and 136 (49\%) received NAC. No gross residual and residual tumor measuring $\leq 1 \mathrm{~cm}$ was achieved in $66 \%$ and $96 \%$ of the PDS group and $79 \%$ and $96 \%$ of the NAC group, respectively. The median progression-free survival (PFS) and overall survival (OS) time were 20 months and not reached, but might be estimated more than 70 months in the PDS group and 15 and 70 months in the NAC group, respectively.

Conclusion: Extensive cytoreductive surgery to minimize residual tumor and selective use of NAC based on the institutional criteria could result in improved survival outcomes. Until further studies can be done to define the selection criteria for NAC after surgery, institutional criteria for NAC should consider the ability of the surgeon and institutional capacity.

Keywords: Ovarian Neoplasms; Neoadjuvant Therapy; Drug Therapy; Debulking Surgical Procedures; Standards 


\section{Author Contributions}

Conceptualization: L.M.C., Y.H.J., S.Y.J., S.S.S., K.S., K.S.H., Y.C.W., P.S.Y.; Data curation: L.M.C., Y.H.J., S.Y.J., S.S.S., K.S., K.S.H., Y.C.W., P.S.Y.; Formal analysis: L.M.C., Y.H.J., S.Y.J., S.S.S., K.S., K.S.H., Y.C.W., P.S.Y.; Investigation: L.M.C. Y.H.J., S.Y.J., S.S.S., K.S., K.S.H., Y.C.W., P.S.Y.; Methodology: L.M.C., Y.H.J., S.Y.J., S.S.S., K.S., K.S.H., Y.C.W., P.S.Y.; Project administration: L.M.C., Y.H.J., S.Y.J., S.S.S., K.S., K.S.H., Y.C.W., P.S.Y.; Resources: L.M.C., Y.H.J., S.Y.J., S.S.S., K.S., K.S.H., Y.C.W., P.S.Y.; Software: L.M.C., Y.H.J., S.Y.J., S.S.S., K.S., K.S.H., Y.C.W., P.S.Y.; Writing - original draft: L.M.C.; Writing - review \& editing: L.M.C., Y.H.J., S.Y.J., S.S.S., K.S., K.S.H., Y.C.W., P.S.Y.

\section{INTRODUCTION}

In the United States, 22,280 new cases and 14,240 deaths of ovarian cancer were estimated in 2016 [1]. The 5-year relative survival rate for ovarian cancer diagnosed between 2005 and 2011 was $92 \%, 73 \%$, and $28 \%$ for localized, regional, and distant disease [1]. In Korea, 2,450 new cases and 1,097 deaths are confirmed according to the Korea Central Cancer Registry [2]. Crude incidence and mortality rates of ovarian cancer are 9.6 and 6.4 per 100,000 females [2]. The annual percent change in incidence of ovarian cancer and epithelial ovarian cancer in Korea is $+1.5 \%$ and $+2.5 \%$ in females, respectively $[3,4]$. The absence of early warning signs or symptoms of ovarian cancer and the lack of screening make conquering the disease challenging. Additionally, more than half of patients were diagnosed at advanced stages.

Primary cytoreductive surgery with adjuvant taxane-platinum chemotherapy is the standard treatment for advanced ovarian cancer. The most important prognostic factor for ovarian cancer is the extent of completeness of cytoreduction [5,6]. The European Organization for Research and Treatment of Cancer-National Cancer Institute of Canada Clinical Trials Group (EORTCNCIC) randomized trial concluded "non-inferior overall survival (OS) rates" of neoadjuvant chemotherapy (NAC) followed by interval debulking and adjuvant chemotherapy compared to primary cytoreductive surgery followed by adjuvant chemotherapy [7]. However, the rate of optimal debulking in terms of residual tumor less than $1 \mathrm{~cm}(42 \%$ for primary cytoreductive group and $81 \%$ for NAC group) is quite low in the trial $[7,8]$. Median progression-free survival (PFS) and OS were 12 and 29 months for the primary cytoreductive surgery group and 12 and 30 months for the NAC group, respectively [7]. In the primary chemotherapy versus primary surgery for newly diagnosed advanced ovarian cancer (CHORUS) trial, median PFS and OS were 11 and 23 months for the primary cytoreductive surgery group and 12 and 24 months for the NAC group, respectively with the rate of optimal debulking in terms of residual tumor less than $1 \mathrm{~cm}$ (41\% for primary cytoreductive group and $73 \%$ for NAC group) [9].

Chi et al. [10] reported the treatment outcomes with same criteria of EORTC trial during same period. A majority of the patients $(90 \%)$ underwent primary cytoreductive surgery in the study. Median PFS and OS were 17 and 50 months, respectively. Recently, NAC guidelines for advanced peritoneal, epithelial ovarian, and fallopian tubal cancer by the Society of Gynecologic Oncology and the American Society of Clinical Oncology suggest that NAC should be individualized according to surgical outcomes including postoperative morbidity and residual tumor [11]. The recommendation is based on the fact that surgical outcomes related to the extent of residual tumor differ among surgeons and across institutions [11-13]. Therefore, treatment outcomes based on institutional criteria for NAC should be investigated further.

Compared to the previous 2 studies [7,10], the National Cancer Center-Korea has used different criteria mainly based on computerized tomography (CT) imaging. The objective of the study was to investigate the treatment outcomes of patients with bulky stage IIIC and IV ovarian cancer who underwent extensive primary debulking surgery (PDS) and selective use of NAC at our institution, using the same inclusion criteria as the EORTC-NCIC trial.

\section{MATERIALS AND METHODS}

After obtaining Institutional Review Board approval (NCCNCS-12-555), medical records were retrospectively reviewed. Patients with bulky stage IIIC and IV ovarian, tubal, and primary 
peritoneal cancer, based on the 1998 International Federation of Gynecology and Obstetrics (FIGO) classification, between December 2000 and March 2009 were included [14]. Metastasis only in the retroperitoneal lymph node (LN) disease was excluded.

The residual tumor has been defined as follows: 1) no gross residual (NGR), reflecting complete cytoreduction to a visibly disease-free state (microscopic residual); 2) gross residual -1 (GR-1), indicating residual disease measuring $\leq 1 \mathrm{~cm}$ in maximal diameter; and 3) gross residual-bulky (GR-B), for residual disease measuring $>1 \mathrm{~cm}$ in maximal diameter [15]. Gross residual (GR) includes GR-1 and GR-B.

The indication of NAC has been described in Table 1. The preoperative evaluation including cytology, pathology, and image were discussed at the tumor board conference by a multidisciplinary team including gynecologic oncologist, surgical oncologist, radiologist, nuclear medicine physician, and pathologist. Tumors at the pelvic cavity were not considered for determination of resectability in general. Tumors at challenging areas such as the smallbowel mesentery, lesser peritoneal sac, porta hepatis, suprarenal LN, or retrocrural LN were considered for resection and discussed extensively by the surgical team during the tumor board [16]. All operations were performed by gynecologic oncologists leading comprehensive surgical teams. The principles of cytoreductive surgical procedures have been described in a report by Lim et al. [17]. Dual thromboprophylaxis of elastic stockings with low-molecularweight heparin were used to prevent postoperative venous thromboembolism [18].

During the cytoreductive surgery, all traces of regressed tumor after NAC were removed as much as possible based on an initial image study to remove potential ovarian cancer stem cells [19]. The abdominal wall was closed layer by layer [20,21]. After cytoreductive surgery, women with ovarian cancer were treated with adjuvant paclitaxel and carboplatin chemotherapy usually for 3-6 cycles depending on the primary treatment, NAC or PDS and pathological response in case of NAC. Less than 10 patients received intraperitoneal chemotherapy (Gynecologic Oncology Group [GOG] 172): most of the patients received less than 1 cycle of intraperitoneal chemotherapy. Some of the patients received hyperthermic intraperitoneal chemotherapy (HIPEC) as part of a phase II and randomized trial [22,23]. Because both studies are ongoing and follow-up has not been completed, HIPEC was not evaluated or analyzed in this study. Posttreatment surveillance included imaging studies such as sonography, CT, magnetic resonance imaging (MRI), or positron emission tomography (PET)/CT and serum CA125 every 3 months for $2-3$ years and then every

Table 1. Current indication of NAC *

\begin{tabular}{l} 
1) Biopsy proven advanced peritoneal, epithelial ovarian, and fallopian tubal cancers or cytology-confirmed \\
adenocarcinoma with negative colonoscopy and esophagogastroduodenoscopy \\
2) Poor performance status and/or grave medical condition, considering intraperitoneal tumor status \\
3) CT findings \\
$\square$ Extra-peritoneal disease (except isolated malignant pleural effusion or resectable metastatic lesions \\
such as cardiophrenic LN metastasis) ${ }^{\dagger}$ \\
$\square$ Involvement of the mesenteric root of the small intestine \\
$\square$ Multiple liver metastases from hematogenous metastasis requiring total resection of liver ${ }^{\ddagger}$ \\
$\square$ Pancreatic metastasis (except pancreatic tail) \\
$\square$ Involvement of the porta hepatis and para-aortic LN metastasis above the renal veins ${ }^{\dagger}$ \\
$\square$ Disease that is larger than 2 cm and perforates the diaphragm ${ }^{\S}$ \\
\hline CT, computerized tomography; LN, lymph node; NAC, neoadjuvant chemotherapy. \\
${ }^{*}$ Adapted from Lim et al. [19]; ${ }^{\dagger}$ Selectively used, considering patient general condition and associated peritoneal \\
tumor burden, especially in the visceral peritoneum; ${ }^{\ddagger}$ Hepatic metastasis from peritoneal seeding is not considered \\
primarily to NAC irrespective of tumor size in most cases except early period [25]; ${ }^{\S}$ Mainly used until the year of 2006.
\end{tabular}


6 months for 2-3 additional years. After 5-years surveillance, serum CA125 levels were checked annually.

Statistical analysis was performed by using the R statistical software version 2.12.1 ( $\mathrm{R}$ Foundation for Statistical Computing, Vienna, Austria; ISBN 3-900051-07-0; http://R-project. org). PFS and OS were calculated from the day of initial treatment to the day of clinical recurrence by CA125 and/or imaging such as CT, MRI, or PET/CT and death, respectively. Survival was estimated with the Kaplan-Meier method and statistical significance was determined by the log-rank test. All p-values presented are 2-sided, and associations are considered significant if the $\mathrm{p}$-value is $<0.05$.

\section{RESULTS}

Of the 316 patients identified with stage III/IV ovarian cancer, 24 patients with stage IIIA and IIIB, and 13 patients with stage IIIC due to isolated LN metastasis were excluded (Fig. 1). Of the remaining 279 patients with bulky stage III/IV, 143 patients $(51 \%)$ were treated with PDS and 136 patients (49\%) were treated with NAC.

Patient characteristics among NCC, Memorial Sloan Kettering Cancer Center (MSKCC), and the EORTC trial are described in Table 2. Median age at NCC was 53 years (range, 26-83 years) and 57 years (range, 26-79 years) for the PDS and NAC groups, respectively. Median age at NCC seems to be lower than that of MSKCC ( 60 years) and the EORTC trial (62 and 63 years for the

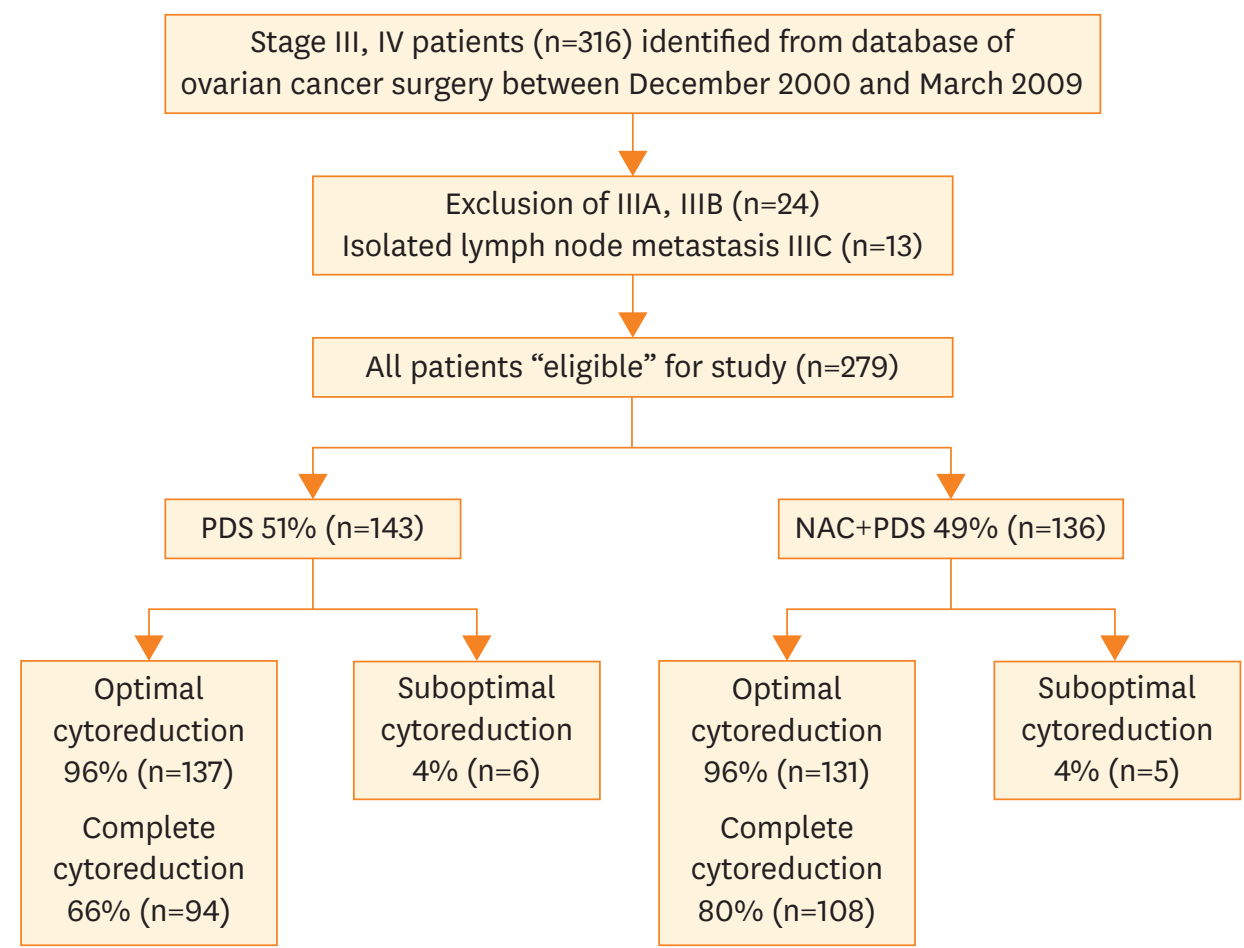

Fig. 1. A flowchart for including patients in the current study. Of the 316 patients with stage III and IV, 279 patients were included. Fifty-one percent (143/279) of the patients were treated with PDS and 49\% (136/279) of the patients were treated with NAC.

NAC, neoadjuvant chemotherapy; PDS, primary debulking surgery. 
Table 2. Patient characteristics for bulky stage IIIC and IV epithelial ovarian cancer

\begin{tabular}{|c|c|c|c|c|c|}
\hline Variables & $\begin{array}{l}\text { PDS arm of NCC } \\
\qquad(n=143)\end{array}$ & $\begin{array}{l}\text { NAC arm of NCC } \\
(n=136)\end{array}$ & $\begin{array}{l}\text { MSKCC } \\
(n=285)\end{array}$ & $\begin{array}{l}\text { PDS arm of EORTC trial } \\
\qquad(n=336)\end{array}$ & $\begin{array}{l}\text { NAC arm of EORTC trial } \\
(n=334)\end{array}$ \\
\hline Age (yr) & $53(26-83)$ & $57(26-79)$ & $60(25-88)$ & $62(25-86)$ & $63(33-81)$ \\
\hline \multicolumn{6}{|l|}{ FIGO stage } \\
\hline IIIC & $115(80.4)$ & $93(68.4)$ & $249(87.0)$ & $257(76.5)$ & $253(75.7)$ \\
\hline IV & $28(19.6)$ & $43(31.6)$ & $36(13.0)$ & 77 (25.9) & $81(24.3)$ \\
\hline Others & $0(0.0)$ & $0(0.0)$ & $0(0.0)$ & $2(0.6)$ & $0(0.0)$ \\
\hline \multicolumn{6}{|l|}{ Tumor grade } \\
\hline 1 & $7(4.9)$ & $8(5.9)$ & $7(2.5)$ & $14(4.2)$ & $10(3.0)$ \\
\hline II & 33 (23.0) & $58(4.3)$ & $34(12.0)$ & $57(17.0)$ & $41(12.3)$ \\
\hline III & $93(65.0)$ & $51(38.0)$ & $237(83.0)$ & $145(43.2)$ & $130(38.9)$ \\
\hline Unknown & $5(3.5)$ & $19(14.0)$ & $7(2.5)$ & $120(35.7)$ & $153(45.8)$ \\
\hline \multicolumn{6}{|l|}{ Histology } \\
\hline Serous & 114 (79.7) & $99(72.8)$ & $249(87.0)$ & $220(65.5)$ & $194(58.1)$ \\
\hline Endometrioid & $5(3.5)$ & $1(0.7)$ & - & $11(3.3)$ & $5(1.5)$ \\
\hline Clear & $2(1.4)$ & $9(6.6)$ & - & $6(1.8)$ & $4(1.2)$ \\
\hline Mucinous & $5(3.5)$ & $1(0.7)$ & - & $8(2.4)$ & $11(3.3)$ \\
\hline Mixed & $8(5.6)$ & $6(4.4)$ & - & $3(0.9)$ & $0(0.0)$ \\
\hline Others & $9(6.3)$ & $20(14.7)$ & $36(13.0)$ & $19(5.7)$ & $30(9.0)$ \\
\hline CA125 (U/dL) & $796(10-20,900)$ & $176(11-41,280)$ & $610(16-14,512)$ & $1,130(16-27,185)$ & $1,180(15-41,456)$ \\
\hline \multicolumn{6}{|c|}{ Upper abdominal procedures } \\
\hline Yes & $111(78.0)$ & $92(68.0)$ & $93(33.0)$ & - & - \\
\hline No & $32(22.0)$ & $44(32.0)$ & $192(67.0)$ & - & - \\
\hline \multicolumn{6}{|l|}{ Residual tumor } \\
\hline Microscopic & $94(65.7)$ & $108(79.4)$ & $69(24.0)$ & $61(19.4)$ & $151(51.2)$ \\
\hline$\leq 1 \mathrm{~cm}$ & $43(30.1)$ & $23(16.9)$ & $134(47.0)$ & $70(22.2)$ & $87(29.5)$ \\
\hline$>1 \mathrm{~cm}$ & $6(4.2)$ & $5(3.7)$ & $82(29.0)$ & $167(53.0)$ & $52(17.7)$ \\
\hline Missing & $0(0.0)$ & $0(0.0)$ & $0(0.0)$ & $17(5.4)$ & $5(1.7)$ \\
\hline
\end{tabular}

Values are presented as median (range) or number (\%). This work was supported by the grant (NCC1610070) of the National Cancer Center, Korea.

EORTC, European Organization for Research and Treatment of Cancer; FIGO, International Federation of Gynecology and Obstetrics; MSKCC, Memorial Sloan Kettering Cancer Center; NAC, neoadjuvant chemotherapy; NCC, National Cancer Center; PDS, primary debulking surgery.

PDS and NAC groups). Stage IIIC composed of $80.4 \%$ and $68.4 \%$ of the patients of the PDS and NAC groups, respectively. More stage IV disease was included in NCC and the EORTC trial ( $25.9 \%$ and $24.3 \%$ of the PDS and NAC groups, respectively) compared to MSKCC (13\%). Serous histology was $80 \%$ and $72 \%$ for the PDS and NAC groups, respectively. Serous histology was included more frequently in NCC and MSKCC (87\%) compared to the EORTC trial $(65.5 \%$ and $58.1 \%$ of the PDS and NAC groups, respectively). Median CA125 was 796 U/dL (range, 10-20; 900 $\mathrm{U} / \mathrm{dL}$ ) and 1,609 U/dL (range, 11-41; $280 \mathrm{U} / \mathrm{dL}$ ) for the PDS and NAC groups, respectively. Upper abdominal surgical procedures were performed in $111(78 \%)$ and $92(68 \%)$ patients in the PDS and NAC groups, respectively. Finally, NGR were observed in 94 (66\%) and 108 (79\%) patients in the PDS and NAC groups, respectively. The percentage of patients with residual tumor $\leq 1 \mathrm{~cm}$ was $96 \%$ and $96 \%$ of the PDS and NAC groups, respectively. Of the women undergoing PDS and NAC, $12.5 \%$ (17/136) and 14.7\% (21/143) were registered in HIPEC clinical trials.

The median PFS was 20 months for the PDS group and 15 months for the NAC group (Fig. 2). The 5 -year PFS was $23 \%$ for PDS and $16 \%$ for NAC ( $\mathrm{p}=0.033)$. The median OS did not reach but more than 70 months for the PDS group and was 70 months for the NAC group. The 5 -year OS was $61 \%$ for PDS and 51\% for NAC ( $\mathrm{p}=0.252)$.

The median PFS was 19 months and 15 months for NGR and GR, respectively (Fig. 3). The 5 -year PFS was $21 \%$ and $17 \%$ for NGR and GR, respectively ( $p=0.069$ ). The median OS did not reach and was 58 months for NGR and GR, respectively. The 5-year OS was $62 \%$ and $45 \%$ for NGR and GR, respectively ( $\mathrm{p}=0.035)$. 

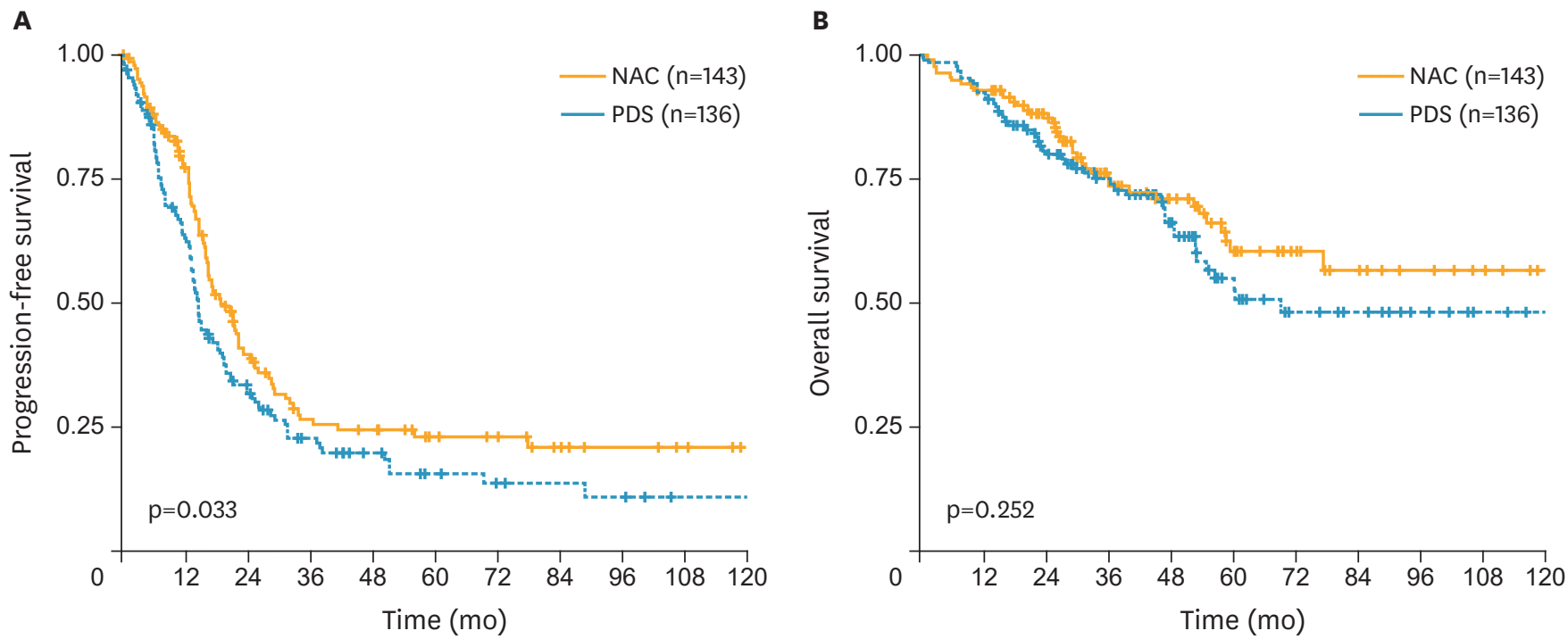

Fig. 2. Survival outcome after extensive cytoreductive surgery and chemotherapy in bulky stage IIIC and IV epithelial ovarian cancer in PDS (blue line) group and NAC (orange line) group. (A) PFS. (B) OS.

OS, overall survival; NAC, neoadjuvant chemotherapy; PDS, primary debulking surgery; PFS, progression-free survival.
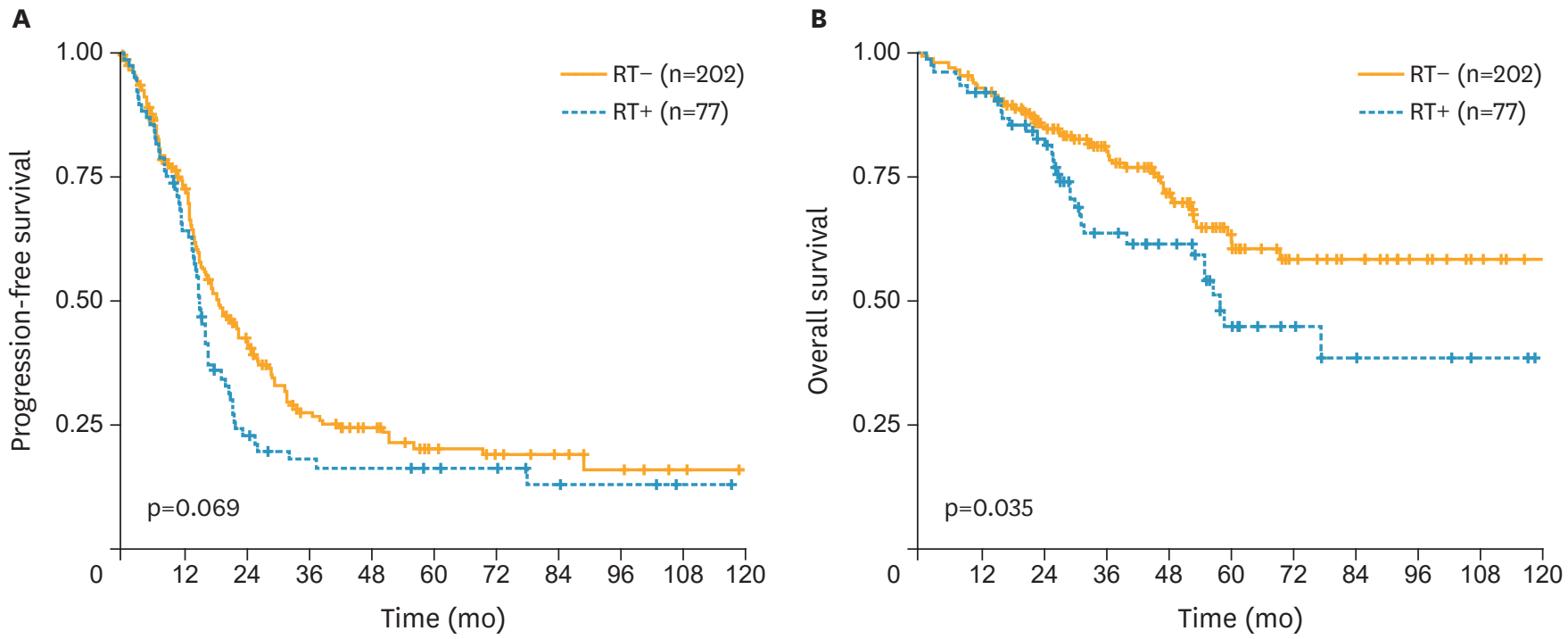

Fig. 3. Survival outcome after extensive cytoreductive surgery and chemotherapy in bulky stage IIIC and IV epithelial ovarian cancer according to microscopic (orange line) and macroscopic (blue line) RT. (A) PFS. (B) OS.

OS, overall survival; PFS, progression-free survival; RT, residual tumor.

\section{DISCUSSION}

Survival outcome in ovarian cancer is significantly correlated with efforts to minimize residual disease, which is successfully achieved by incorporating upper abdominal surgical procedures $[7,10,18]$. This is consistent with previous meta-analysis for a positive correlation between increased median OS with an increased rate of optimal debulking [6]. The survival benefit of an increased rate of complete or optimal cytoreductive surgery in the management of ovarian cancer was observed in the current study and previous studies $[6,15,24]$. 
In the current study, the indication for NAC (Fig. 1) was changed as surgical skills and instruments improved [11]. We recently reported feasible cytoreduction in challenging areas including the liver, porta hepatis, and cardiophrenic LN metastases [25-28]. The indication for NAC might change with the increasing rate of complete or optimal cytoreduction. The indication for NAC is based on hospital capacity, multi-disciplinary surgical teams, and, mostly, the skill and experience of the surgeon. We believe that primary cytoreductive surgery to minimize the residual tumor which does not delay the postoperative chemotherapy resulting NGR is important. If macroscopic residual tumor after cytoreductive surgery is expected on the challenging surgical area preoperatively, NAC could be used as an alternative treatment.

Some explanation might be needed for the significantly improved survival rates in the current study compared to previous studies $[7,9,10]$. First, better OS definitively comes from higher rates of upper abdominal surgical procedures resulting in higher rates of complete or optimal debulking. Even within the same NGR or GR-1 groups, the volume of the residual tumor was quite different according to the surgical effort. Recently, our surgical team has tried to palpate and remove all metastatic lesions, including challenging areas such as the porta hepatis or the lesser peritoneal sac [27]. In the previous study, increased likelihood of cytoreduction and risk reduction of death in hospital is estimated in high-volume hospital [13]. Second, regular surveillance for recurrence using image studies such as CT, MRI, or PET/CT after completion of the primary treatment irrespective of symptoms or signs suggestive of recurrence, or elevation of CA125 might offer an opportunity to undergo curative treatment including a secondary cytoreductive surgery and/or chemotherapy. In Korea, women with ovarian cancer received a PET/CT if there was clinical suspicion of recurrence at any time with National Health Insurance. The cost of PET/CT for the women with ovarian cancer was about 40 USD, excluding reimbursement from the National Health Insurance system. Inexpensive early detection and intervention might be one explanation for the survival improvement of these patients. Third, several clinical trials including HIPEC might be one of the reasons for the improved survival outcomes [22]. Fourth, relatively short follow-up interval might be another reason for the improved survival outcomes. Fifth, removing all traces of regressed tumor after NAC may have reduced the residual ovarian cancer stem cells [19]. Lastly, some of the women who received NAC and showed a poor response did not undergo interval debulking surgery and were excluded for this report. This may be another explanation from the current results. For a proper comparison based on an intention-to-analysis, patients who received chemotherapy only should be included. In such a subgroup, patients with poor responses to NAC and patients with excellent responses, such as complete response based on imaging and CA125, might be included. These clinical situations have not been considered in most of the previous studies and careful comparison and interpretation is required.

Although they have the same rate of complete or optimal debulking, poor survival outcomes might be expected in the NAC group compared to the PDS group [7]. This might be related with the possibility of higher concentration of platinum- and/or taxane-resistant ovarian cancer cells, including ovarian cancer stem cells in same bulk tumor after NAC $[8,17]$. Our surgical policy after NAC, "removing all traces of regressed tumor after NAC," needs to be confirmed in future studies.

We believe that the criteria for NAC should be individualized based on surgeon experience, hospital capacity, and the cooperation of surgeons from other departments [12,13,29,30]. The criteria changed with the evolution of surgical experience and technique [11]. Therefore, 
the criteria for NAC suggested in the table are typical, not absolute, for the most of the cases in this study.

The weakness of the current study is the retrospective study design, potentially including selection bias. The definition for each variable might be different across the studies compared because of the design of each study. The comparable and consistent definition of variables is needed for the conclusion based on the rational comparison. Furthermore, some of the women who received NAC might be excluded in the current analysis. This study was based on the per-protocol analysis: primary surgery vs. NAC followed by interval debulking surgery. The intention-to-treatment (ITT) analysis might reveal different results. Till now, ITT like 2 randomized trials has not been analyzed from the institutional experiences $[7,9]$.

In conclusion, extensive cytoreductive surgery to minimize residual tumor and selective use of NAC based on the institutional criteria could result in improved survival outcomes. Until further studies can be done to define and improve the selection criteria for NAC, institutional criteria for NAC should currently consider the ability of the surgeon and institutional capacity for patients who may benefit from NAC.

\section{REFERENCES}

1. Siegel RL, Miller KD, Jemal A. Cancer statistics, 2016. CA Cancer J Clin 2016;66:7-30. PUBMED | CROSSREF

2. Jung KW, Won YJ, Oh CM, Kong HJ, Cho H, Lee JK, et al. Prediction of cancer incidence and mortality in Korea, 2016. Cancer Res Treat 2016;48:451-7. PUBMED | CROSSREF

3. Lim MC, Moon EK, Shin A, Jung KW, Won YJ, Seo SS, et al. Incidence of cervical, endometrial, and ovarian cancer in Korea, 1999-2010. J Gynecol Oncol 2013;24:298-302. PUBMED | CROSSREF

4. Kim SI, Lim MC, Lim J, Won YJ, Seo SS, Kang S, et al. Incidence of epithelial ovarian cancer according to histologic subtypes in Korea, 1999 to 2012. J Gynecol Oncol 2016;27:e5. PUBMED | CROSSREF

5. Bristow RE, Puri I, Chi DS. Cytoreductive surgery for recurrent ovarian cancer: a meta-analysis. Gynecol Oncol 2009;112:265-74.

PUBMED | CROSSREF

6. Bristow RE, Tomacruz RS, Armstrong DK, Trimble EL, Montz FJ. Survival effect of maximal cytoreductive surgery for advanced ovarian carcinoma during the platinum era: a meta-analysis. J Clin Oncol 2002;20:1248-59. PUBMED | CROSSREF

7. Vergote I, Tropé CG, Amant F, Kristensen GB, Ehlen T, Johnson N, et al. Neoadjuvant chemotherapy or primary surgery in stage IIIC or IV ovarian cancer. N Engl J Med 2010;363:943-53. PUBMED | CROSSREF

8. Lim MC, Park SY. Standardization of cytoreductive surgery is a precondition for clinical trials of ovarian cancer including cytoreductive surgery and/or neoadjuvant chemotherapy. Gynecol Oncol 2012;125:510. PUBMED | CROSSREF

9. Kehoe S, Hook J, Nankivell M, Jayson GC, Kitchener H, Lopes T, et al. Primary chemotherapy versus primary surgery for newly diagnosed advanced ovarian cancer (CHORUS): an open-label, randomised, controlled, non-inferiority trial. Lancet 2015;386:249-57. PUBMED | CROSSREF

10. Chi DS, Musa F, Dao F, Zivanovic O, Sonoda Y, Leitao MM, et al. An analysis of patients with bulky advanced stage ovarian, tubal, and peritoneal carcinoma treated with primary debulking surgery (PDS) during an identical time period as the randomized EORTC-NCIC trial of PDS vs neoadjuvant chemotherapy (NACT). Gynecol Oncol 2012;124:10-4.

PUBMED | CROSSREF 
11. Vergote IB, Van Nieuwenhuysen E, Vanderstichele A. How to select neoadjuvant chemotherapy or primary debulking surgery in patients with stage IIIC or IV ovarian carcinoma. J Clin Oncol. Forthcoming 2016. PUBMED

12. Bristow RE, Puri I, Diaz-Montes TP, Giuntoli RL, Armstrong DK. Analysis of contemporary trends in access to high-volume ovarian cancer surgical care. Ann Surg Oncol 2009;16:3422-30. PUBMED | CROSSREF

13. Bristow RE, Zahurak ML, Diaz-Montes TP, Giuntoli RL, Armstrong DK. Impact of surgeon and hospital ovarian cancer surgical case volume on in-hospital mortality and related short-term outcomes. Gynecol Oncol 2009;115:334-8. PUBMED | CROSSREF

14. Odicino F, Pecorelli S, Zigliani L, Creasman WT. History of the FIGO cancer staging system. Int J Gynaecol Obstet 2008;101:205-10.

PUBMED | CROSSREF

15. Chang SJ, Bristow RE. Evolution of surgical treatment paradigms for advanced-stage ovarian cancer: redefining 'optimal' residual disease. Gynecol Oncol 2012;125:483-92. PUBMED | CROSSREF

16. Choi HJ, Lim MC, Bae J, Cho KS, Jung DC, Kang S, et al. Region-based diagnostic performance of multidetector CT for detecting peritoneal seeding in ovarian cancer patients. Arch Gynecol Obstet 2011;283:353-60.

PUBMED | CROSSREF

17. Lim MC, Seo SS, Kang S, Kim SK, Kim SH, Yoo CW, et al. Intraoperative image-guided surgery for ovarian cancer. Quant Imaging Med Surg 2012;2:114-7. PUBMED

18. Lim MC, Lee HS, Kang S, Seo SS, Lee BY, Park SY. Minimizing tumor burden by extensive cytoreductive surgery decreases postoperative venous thromboembolism in ovarian clear cell carcinoma. Arch Gynecol Obstet 2010;281:329-34. PUBMED | CROSSREF

19. Lim MC, Song YJ, Seo SS, Yoo CW, Kang S, Park SY. Residual cancer stem cells after interval cytoreductive surgery following neoadjuvant chemotherapy could result in poor treatment outcomes for ovarian cancer. Onkologie 2010;33:324-30. PUBMED | CROSSREF

20. Kim SI, Lim MC, Song YJ, Seo SS, Kang S, Park SY. Application of a subcutaneous negative pressure drain without subcutaneous suture: impact on wound healing in gynecologic surgery. Eur J Obstet Gynecol Reprod Biol 2014;173:94-100. PUBMED | CROSSREF

21. Kim SI, Lim MC, Bae HS, Shin SR, Seo SS, Kang S, et al. Benefit of negative pressure drain within surgical wound after cytoreductive surgery for ovarian cancer. Int J Gynecol Cancer 2015;25:145-51. PUBMED | CROSSREF

22. Lim MC, Kang S, Choi J, Song YJ, Park S, Seo SS, et al. Hyperthermic intraperitoneal chemotherapy after extensive cytoreductive surgery in patients with primary advanced epithelial ovarian cancer: interim analysis of a phase II study. Ann Surg Oncol 2009;16:993-1000. PUBMED | CROSSREF

23. Park SY. Intraoperative hyperthermic intraperitoneal chemotherapy with ovarian cancer. Bethesda (MD): Clinical-Trials.gov; 2010.

24. Chi DS, Eisenhauer EL, Zivanovic O, Sonoda Y, Abu-Rustum NR, Levine DA, et al. Improved progressionfree and overall survival in advanced ovarian cancer as a result of a change in surgical paradigm. Gynecol Oncol 2009;114:26-31. PUBMED | CROSSREF

25. Lim MC, Kang S, Lee KS, Han SS, Park SJ, Seo SS, et al. The clinical significance of hepatic parenchymal metastasis in patients with primary epithelial ovarian cancer. Gynecol Oncol 2009;112:28-34. PUBMED | CROSSREF

26. Lim MC, Lee HS, Jung DC, Choi JY, Seo SS, Park SY. Pathological diagnosis and cytoreduction of cardiophrenic lymph node and pleural metastasis in ovarian cancer patients using video-assisted thoracic surgery. Ann Surg Oncol 2009;16:1990-6. PUBMED | CROSSREF

27. Song YJ, Lim MC, Kang S, Seo SS, Kim SH, Han SS, et al. Extended cytoreduction of tumor at the porta hepatis by an interdisciplinary team approach in patients with epithelial ovarian cancer. Gynecol Oncol 2011;121:253-7.

PUBMED | CROSSREF 
28. Yoo HJ, Lim MC, Song YJ, Jung YS, Kim SH, Yoo CW, et al. Transabdominal cardiophrenic lymph node dissection (CPLND) via incised diaphragm replace conventional video-assisted thoracic surgery for cytoreductive surgery in advanced ovarian cancer. Gynecol Oncol 2013;129:341-5. PUBMED | CROSSREF

29. Bristow RE, Chang J, Ziogas A, Randall LM, Anton-Culver H. High-volume ovarian cancer care: survival impact and disparities in access for advanced-stage disease. Gynecol Oncol 2014;132:403-10. PUBMED | CROSSREF

30. Kang S, Jong YH, Hwang JH, Lim MC, Seo SS, Yoo CW, et al. Is neo-adjuvant chemotherapy a "waiver" of extensive upper abdominal surgery in advanced epithelial ovarian cancer? Ann Surg Oncol 2011;18:3824-7. PUBMED | CROSSREF 